\title{
怒江河谷栗喉蜂虎的繁殖行为观察
}

\author{
吴忠荣 ${ }^{1,2}$, 韩联宪 ${ }^{1, *}$, 匡中帆 ${ }^{1,2}$ \\ （1. 西南林学院 保护生物学学院 云南 昆明 650224；2. 贵阳市黔灵公园管理处 贵州 贵阳 550003）
}

\begin{abstract}
摘要: 2007 年 3 月 26 日-7 月 16 日, 采用典型野外记录法和所有事件取样法对云南省保山市道街怒江河谷 栗喉蜂虎繁殖行为进行了观察。结果表明：1）产卵期雄性栗喉蜂虎求偶喂食的食物以蜻蜓目和膜翅目昆虫为主, 占 $83.56 \%$; 雌鸟接受喂食的可能性为 96.83\%, 接受喂食后仅有 $17.49 \%$ 的雌鸟与雄鸟进行交配。2）在孵卵期昼 间，雄鸟每次坐巢的时间为（23.5 $\pm 21.6 ） \mathrm{~min}$, 每日坐巢的时间占 $54.4 \%$; 雌鸟每次坐巢的时间为 $(25.8 \pm 11.5$ ) $\min$, 每日坐巢的时间占 42.1\%, 雌雄鸟每次坐巢的时间无明显差异。3）幼倠出壳后亲鸟对幼雏的暖雉时间逐日 减少, 至第 $20 \mathrm{~d}$ 亲鸟不再暖雉。栗喉蜂虎的育倠期为 $29 \sim 33 \mathrm{~d}$, 在繁殖过程中帮助喂食的鸟确实能提高被助鸟的 繁殖成功率。
\end{abstract}

关键词: 栗喉蜂虎; 繁殖行为; 怒江河谷

中图分类号: Q959.737; Q959.737.08 文献标识码：A 文章编号：0254-5853-(2009)04-0429-04

\section{Breeding Behaviors of Blue Tailed Bee-eater of Nujiang Valley}

\author{
WU Zhong-rong ${ }^{1,2}$; HAN Lian-xian ${ }^{1, *}$; KUANG Zhong-fan ${ }^{1,2}$ \\ (1. Faculty of Conservation Biology, Southwest Forestry University, Kunming, 650224, China; \\ 2. Qianling Park of Guiyang, Guiyang, Guizhou 550003, China)
}

\begin{abstract}
By the method of ad libitum sampling and all-occurrence recording, breeding behaviors of Blue Tailed Bee-eater in the Agricultural Region of Nujiang Valley were observed from 26th March to 17th July, 2007. The result showed the most common foods that male birds fed to female birds are Odonata and Hymenoptera insects, accounting for $83.56 \%$. The frequency of females accepting the food provided by males was $96.83 \%$, and the frequency of copulation was $17.49 \%$ following food acceptance. During the daytime of the incubation period, comparison of time on nest between male and female showed no significant difference. Male mean on nest time was (23.5 \pm 21.6$)$ min and female (25.8 \pm 11.5$) \mathrm{min}$. Once hatched, a decrease in time spent warming the young was seen until the 20th day where the parents bird ceased warming. The nursing period of Blue Tailed Bee-eater was found to be 29 - 33 days, with the presence of a helper significantly improving the success rate of reproduction.
\end{abstract}

Key words: Blue Tailed Bee-eater; Breeding Behavior; Nujiang Valley

栗喉蜂虎(Merops philippinus)隶属佛法僧目 (Coraciformes) 蜂虎科 (Meropidae)。在中国分布于 云南西部至东南部、四川西南部、广西北部湾、广 东沿海 (夏候鸟) 以及海南 (留鸟), 国外分布于 印度、东南亚、南亚, 南至印度尼西亚, 东至菲律 宾及伊里安岛 (Tan \& Guan, 2003)。对栗喉蜂虎的 研究较少, 台湾学者在金门岛对栗喉蜂虎的巢地复 育效应与栖息地选择模式、生殖生态学、单独和集
群营巢的生殖策略进行过研究，认为栗喉蜂虎偏好 于沙土土质，且选择朝南偏东方的断崖面筑巢，有 单独营巢与集群营巢两种策略(Wang, 2005; Wang \& Yuan, 2005; Wang, 2005; Yuan et al, 2006)。 国外曾报道过栗喉蜂虎的种内合作繁殖现象, Brent (2002) 观察了 16 巢栗喉蜂虎的繁殖, 有 8 巢具 有帮助喂食鸟, 且帮助喂食鸟不只帮助一个繁殖对 喂食幼鸟。栗喉蜂虎的求偶喂食行为仅见少量文字 
描述, 而栗喉蜂虎的孵化行为和育雉行为等未见报 道。笔者 2007 年春夏季在云南省保山市道街对栗 喉蜂虎的繁殖行为进行了系统观察, 现将其结果报 道如下。

\section{1 研究地概况和方法}

\section{1 研究地自然概况}

云南保山市怒江河谷属印度洋西南季风区, 干 湿季明显。海拔 670 $1300 \mathrm{~m}$; 地处北纬 $24^{\circ} 59^{\prime} \sim$ $25^{\circ} 01^{\prime}$ ，东经 $98^{\circ} 53^{\prime} \sim 98^{\circ} 57^{\prime}$ 。年太阳总辐 射 $579.70 \mathrm{~kJ} / \mathrm{cm}^{2}$ 。年均温 $21.5^{\circ} \mathrm{C}$, 最冷月均温 13.9 ${ }^{\circ} \mathrm{C}$, 最热月均温 $26.4^{\circ} \mathrm{C}$, 极端最低温 $0.2^{\circ} \mathrm{C}$, 极端 最高温 $40.4^{\circ} \mathrm{C}, \geqslant 10^{\circ} \mathrm{C}$ 积温 $7800^{\circ} \mathrm{C}$ 。年降雨量 751.4 $\mathrm{mm}$; 雨季 (5 月- 10 月) 降水量 $618.6 \mathrm{~mm}$, 占年 降水量的 $82 \%$; 旱季(11月一 4 月)降水量 $132.8 \mathrm{~mm}$, 仅占年降水量的 $18 \%$; 年均干燥度 1.9 。土壤以燥 红壤为主 (Cao, 1993)。种植的农作物主要有玉米

(Sedum dbum)、大豆 (Leguminosea sp.)、番茄 (Lycopersicum esculentum) 等, 经济果树林主要有 龙眼 ( Dimocarpus longan)、香蕉 ( Musa sappientum) 、荔枝 (Litchinenswas sonn)、咖啡 (Coffea congenswas Froehn) 等。研究区域位于保 山坝湾镇道街村东岸沿江农耕区。

\section{2 研究方法}

1.2.1 观察时间 选择栗喉蜂虎始迁入繁殖地至 幼鸟出巢期间进行观察，时间为 2007 年 3 月 26 日
-7 月 16 日。其中 3 月 26 日-4 月 14 日为预观 察, 4 月 15 日一 7 月 16 日为正式观察, 去除天气 恶劣不能工作的时间, 有效工作日 $102 \mathrm{~d}$ 。

1.2.2 营巢点确定和观察方法 栗喉蜂虎在道街 怒江东岸农耕区有 6 个集群营巢点。新田洼子营巢 点为一个不能耕作的 “ $\mathrm{Y}$ ” 字型冲沟, 东南西北四 个方向裸露断崖面均有栗喉蜂虎筑巢, 加上该点栗 喉蜂虎的集群数量较大, 故选择在此营巢点进行繁 殖习性的观察。在营巢地附近搭建隐蔽棚, 借助奥 林巴斯 $10 \times 42$ 双筒望远镜进行观察。

1.2.3 观察鸟标记 栗喉蜂虎雌雄鸟外形相近, 雌 雄难辨。在正式观察前用鸟网捕捉栗喉蜂虎, 使用 章华染发剂对栗喉蜂虎不同部位染色，并用不同颜 色棉质线对其飞羽进行标记; 性别通过标记后的行 为确定。共捕捉并标记栗喉蜂虎 24 只, 其中雄鸟 11 只, 雌鸟 13 只。有 4 个繁殖巢的䧳雄鸟均被标 记，共计标记到 20 个繁殖巢的成鸟。

1.2.4 繁殖行为期的划分 根据各行为发生的不 同时间, 将栗喉蜂虎的繁殖期分为巢址选择期、产 卵期、孵化期、育雉期(各行为时期定义见表 1)。繁 殖行为期的划分以所标记的栗喉蜂虎繁殖个体 $60 \%$ 以上发生的行为为标准, 例如: 所标记的栗喉 蜂虎繁殖个体经常表现出求偶喂食行为, 则定为产 卵期; 产卵期后若 $60 \%$ 以上标记个体进入巢洞孵 卵, 则划为孵化期, 以此类推。在各行为期观察繁 殖行为, 产卵期观察栗喉蜂虎的求偶喂食行为。

表 1 栗喉蜂虎的繁殖行为时期定义

Tab. 1 Description of breeding Period of Blue Tailed Bee-eater

\begin{tabular}{|c|c|}
\hline 繁殖期 Breeding period & 行为特征 Characteristics of behavior \\
\hline 巢址选择期 & \\
\hline Nesting sites selection period & 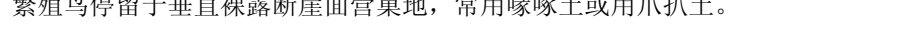 \\
\hline 产卵期 Laying period & $\begin{array}{l}\text { 雄鸟携带食物到营巢地, 雌鸟从巢洞飞出, 雄鸟将处理或未处理的食物递予雌鸟, } \\
\text { 雌鸟接受喂食; 接受喂食后常有交配行为的发生, 雌鸟飞回巢洞。 }\end{array}$ \\
\hline 孵化期 Incubation period & 雌雄鸟不携带食物轮流进入巢洞睬卵。 \\
\hline 育雏期 Nursing period & 雌雄鸟及帮助喂食鸟携带食物进入巢洞喂食幼鸟, 并在洞内对幼维进行保暖。 \\
\hline
\end{tabular}

1.2.5 行为取样方法 采用典型野外记录法, 于 2007 年 5 月 16-23 日观察记录产卵期栗喉蜂虎的 求偶喂食行为, 记录求偶喂食的食物大类、喂食频 次, 雌鸟是否接受喂食以及喂食后是否会有交配行 为。用所有事件取样法 (Xu \& Zhang, 1998), 于 2007 年 5 月 24 日一 6 月 13 日的 7:00-19:00, 观察 雌雄鸟昼间孵卵时间。于 2007 年 6 月 14 日一 7 月 16 日, 用所有事件取样法观察记录育雉期栗喉蜂虎
的暖雉时间以及喂食幼雉的频率。

1.2.6 数据处理方法 收集的数据用 Excel 2003 处 理, 栗喉蜂虎繁殖对雌雄鸟昼间孵卵时间的差异用 SPASS 软件进行单因素方差分析。

\section{2 结果}

\section{1 繁殖行为期}

栗喉蜂虎的繁殖行为时期时间划分见表 2 。 


\section{2 栗喉蜂虎的求偶喂食行为}

野外观察到栗喉蜂虎的求偶喂食行为共计 189

次, 结果如表 3 所示。

\section{3 亲鸟昼间卵孚卵坐巢时间}

对孵卵期昼间 4 个繁殖对的亲鸟共 $12647 \mathrm{~min}$ 的坐巢时间进行观察, 结果表明: 雄鸟每次坐巢时 间为 $(23.5 \pm 21.6) \min (n=276)$, 每日坐巢时间占
$54.4 \%$; 雌鸟每次坐巢时间为 ( $25.8 \pm 11.5$ ) $\min (n=191)$, 每日坐巢时间占 $42.1 \%$; 轮流坐巢时, 雌雄鸟均不在巢内的时间为 (3.4 \pm 2.7$) \mathrm{min}$, 占 2.3\%; 雌雄亲鸟同在巢内的时间为 $(1.5 \pm 0.3) \mathrm{min}$, 占 $1.2 \%$ 。 雌雄鸟昼间坐巢时间无显著差异（ $P=0.685>0.05$, 单因素方差分析检验)。

\section{4 亲鸟暖雏时间比率}

表 2 栗喉蜂虎繁殖行为时期的划分

Tab. 2 Division of breeding period of Blue Tailed Bee-eater

\begin{tabular}{|c|c|}
\hline 繁殖行为期 Breeding period & 时间划分 Date \\
\hline 巢址选择期 Nesting sites selection period & 4 月 15 日一 5 月 19 日 \\
\hline 产卵期 Laying period & 5 月 11 日一 5 月 23 日 \\
\hline 孵化期 Incubation period & 5 月 16 日一 6 月 21 日 \\
\hline 育雉期 Nursing period & 6 月 5 日一 7 月 20 日 \\
\hline
\end{tabular}

表 3 栗喉蜂虎求偶喂食行为的食物组成、接受喂食及交配频率

Tab. 3 Food composition of courtship feeding and mating frequency of Blue Tailed Bee-eater

\begin{tabular}{|c|c|c|c|c|c|c|}
\hline & \multicolumn{4}{|c|}{ 食物种类 Food composition } & \multirow{2}{*}{$\begin{array}{c}\text { 接受喂食 } \\
\text { Accepting food }\end{array}$} & \multirow{2}{*}{$\begin{array}{c}\text { 接受喂食后交配 } \\
\text { Mating }\end{array}$} \\
\hline & $\begin{array}{l}\text { 蜻蜓目 } \\
\text { Odonata }\end{array}$ & $\begin{array}{c}\text { 鳞翅目 } \\
\text { Lepidoptera }\end{array}$ & $\begin{array}{c}\text { 膜翅目 } \\
\text { Hymenoptera }\end{array}$ & 其他 Other & & \\
\hline 次数 Times & 119 & 14 & 39 & 17 & 183 & 32 \\
\hline 频率 Frequency（\%) & 62.96 & 7.41 & 20.6 & 8.99 & 96.83 & 17.49 \\
\hline
\end{tabular}

对 4 个繁殖对的雌雄鸟共计进行了 $11091 \mathrm{~min}$ 的观察, 亲鸟对幼倠的暖雉时间比率见图 1 。栗喉 蜂虎幼倠出壳当日, 亲鸟的暖倠时间比率为 $93.1 \%$; 第 14 天, 暖雉时间比率为 $9.0 \%$; 至第 $20 \mathrm{~d}$, 暖雉 时间比率为 $3.1 \%$ 。

\section{5 栗喉蜂虎给幼维的喂食次数}

对有 4 只幼倠的 7 个繁殖对的栗喉蜂虎进行了 $26 \mathrm{~d}$ 的观察, 共观察到 2005 次对幼倠的喂食行为, 其中帮助喂食鸟喂食 373 次。栗喉蜂虎幼雉出壳当 日共喂食 52.1 次, 第 $17 \mathrm{~d}$ 为 172.3 次, 第 $23 \mathrm{~d}$ 喂食 164.6 次, 第33d喂食 6.5 次 (图 2)。

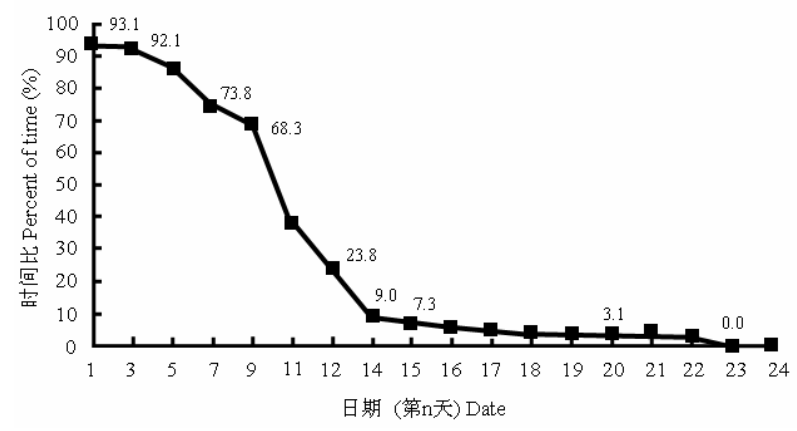

图 1 育雉期栗喉蜂虎的暖雉时间比率

Fig. 1 The percent of time of Blue Tailed Bee-eater warming the fledging bird

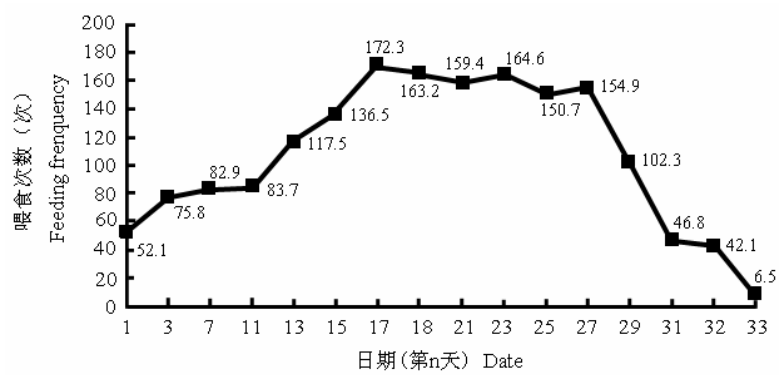

图 2 栗喉蜂虎对幼倠的喂食次数

Fig. 2 The feeding frequency of Blue Tailed Bee-eater

\section{3 讨 论}

\section{1 栗喉蜂虎雄鸟求偶喂食行为}

栗喉蜂虎的求偶喂食行为表现为雄鸟对雌鸟 的喂食以及雌鸟向雄鸟乞食的行为, 雌鸟接受食物 后可能会发生交配行为。产卵期雄鸟喂给雌鸟的食 物主要以蜻蜓目和膜翅目昆虫为主, 两者共占喂食 次数的 $83.56 \%$ 。雄鸟喂给雌鸟食物, 雌鸟虽有 $96.83 \%$ 的可能接受喂食, 但接受喂食后仅有 $17.49 \%$ 的雌鸟与雄鸟交配 (表 3)。此结果与台湾国立中学 许永面的观察有较大差异。他在金门观察发现栗喉 蜂虎雌鸟一旦接受食物, 则立即与雄鸟进行交配

(Xu et al, 1990)。怒江河谷栗喉蜂虎雌岛接受食 物后与雄鸟发生交配行为频率较低的原因, 可能因 
为研究区域地处干热河谷, 营巢点离怒江较近, 怒 江江面有大量昆虫活动。因气候较为炎热, 昆虫繁 殖很快, 为栗喉蜂虎提供了大量的食物, 致使雌鸟 较容易获取食物, 不必以接受交配的方式换取雄岛 的喂食。

\section{2 栗喉蜂虎对幼维的喂食次数}

栗喉蜂虎每窝幼倠数为 2 6 只, 以 4 只幼倠居 多。研究期间对有 4 只幼雏的 7 对栗喉蜂虎的育雉 行为进行了观察。同时, 对有 2 只幼倠的 1 个繁殖 对进行了观察, 结果表明: 幼雉数 2 只的繁殖鸟, 对幼倠的喂食次数仅相当于有 4 只幼维的喂食次数 的 $45.3 \%$ 。

栗喉蜂虎幼倠快离巢时, 亲鸟有呼叫幼倠离巢 的行为。所观察的其中一个繁殖对, 幼踓于 6 月 14 日出壳, 亲鸟在幼唯出壳后第 $30 \mathrm{~d}$ 即 7 月 13 日开 始呼叫幼鸟离巢。7 月 14 日喂食次数与唤幼次数相 近, 分别为 43 次和 47 次; 但 7 月 15 日亲鸟飞到 洞口 99 次, 喂食次数仅为 40 次; 16 日 9:00-10:32 共喂食 3 次, 呼叫次数为 18 次, 远比喂食次数要

\section{参考文献:}

Burt DB. 2002. Social and breeding of Bee-eaters in Thailand [J]. Wilson Bull, 114(2): 275-279.

Cao YH. 1993. The flora of Nujiang Dry-hot valley of Lujiangba of Yunnan[J]. Acta Bot Yunnan, 15(4): 284-287. [曹永恒. 1993. 云南潞 江坝怒江干热河谷植物区系研究. 云南植物研究, 15(4): 284-287.]

Tan YK, Guan GX. 2003. Fauna sinica.: Vol.7. Aves. Coraciformes [M]. Beijing: Science Press, 86-87. [谭耀匡, 关贯勋. 2003. 中国动物志. 回纲一一第七卷: 佛法僧目. 北京: 科学出版社, 86-87.]

Wang YJ, Yuan HW. 2005. Breeding biology of blue-tailed bee-eater (Merops philipennus) in Kinmen Island[C]. The 2005 Animal Behavior and Ecology Annual Conference Proceeding. Taibei. [王元均, 袁孝维. 2005. 金门栗喉蜂虎生殖生态学研究. 动物行为与生态, 2005 年联 合学术年会论文集. 台北.]

Wang YJ. 2005. Analysis of solo-breeding and colony - breeding strategy of blue-tailed bee-eater (Merops philipennus) in Kinmen Island[D]. Master's thesis. Faculty of the Graduate Institute of Forestry National Taiwan University. [王元均. 2005. 金门岛栗喉蜂虎单独与集群营巢 之生殖策略分析. 台湾: 台湾大学森林环境及资源学研究所 (硕士 论文）.]
高。幼鸟分别在第 $32 \mathrm{~d}$ 和第 $33 \mathrm{~d}$ 离巢, 7 月 16 日 10:32 最后 1 只幼维离巢, 育雉期 $33 \mathrm{~d}$ 。而另外 2 只幼维的幼岛, 育雉期为 $29 \mathrm{~d}$ 。

由于此次研究未标记到帮助喂食鸟, 对于研究 区域的栗喉蜂虎帮助喂食鸟是否会如 Brent(2002) 所报道的帮助 1 对以上的成鸟繁殖是个尚待深入研 究的问题。另外, 研究过程中观察到繁殖失败后成 为帮助喂食鸟的成鸟, 也仅观察到其帮助 1 个而并 非多个繁殖对喂食幼鸟的行为, 所喂食的幼鸟离巢 后, 帮助喂食鸟也跟随幼鸟离开营巢地。

\section{3 栗喉蜂虎的其他繁殖习性}

观察发现, 所标记的 20 个繁殖对中, 巢繁殖 成功率为 $65 \%$, 有 $75 \%$ 的栗喉蜂虎繁殖对具有帮助 喂食鸟, 有帮助喂食鸟的巢繁殖成功率达 73.3\%, 无帮助喂食鸟的巢繁殖成功率仅为 $40 \%$ 。这说明在 繁殖过程中帮助喂食鸟的喂食帮助确能提高被助 者的繁殖成功率。另外, 在帮助喂食鸟的 373 次喂 食行为中, 仅观察到对幼雉的喂食, 未发现其有暖 雏行为。

Wang YP. 2005. Effect of breeding colony of restoration and habitat selection model of blue-tailed bee-eater (Merops philipennus) in Kinmen Island[D]. Master's thesis. Faculty of the Graduate Institute of Forestry National Taiwan University. [王怡平. 2005. 金门栗喉蜂虎营 巢地復育效应与栖息地选择模式. 台湾: 台湾大学森林学研究所 (硕士论文).]

Xu HF, Zhang ED. 1998. Wildlife Conservation and Management Principles and Techniques [M]. Shanghai: East China Normal University Press, 69-70. [徐宏发, 张恩迪. 1998. 野生动物保护原理及管理技术. 上 海: 华东师范大学出版社, 69-70.]

Xu YM, Li QF, Li WL. 1990. Study on habitat distribution and breeding behavior of blue-tailed bee-eater (Merops philipennus)[EB/OL]. Kinmen national senior middle school. [许永面, 李庆丰, 李温林. 1990. 栗喉蜂虎的栖地分布与繁殖行为的研究. 台湾: 国立金门高 级中学.]

Yuan HW, Wang MK, Chang WL, Wang LP, Chen YM, Chou CR. 2006. Soil composition affects the nesting behavior of blue-tailed bee-eaters (Merops philippinus) on Kinmen Wasland [J]. Ecol Res, 21(4): 510-512. 